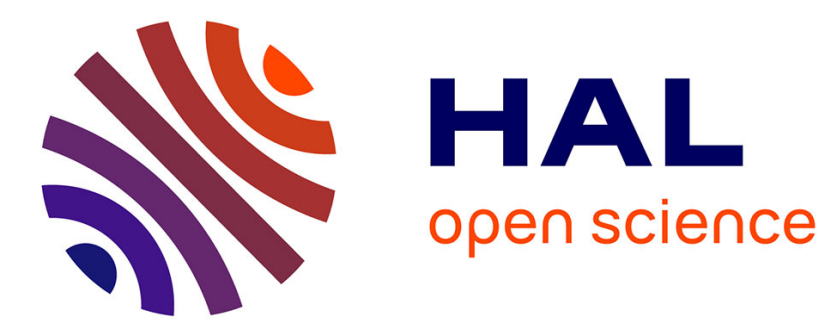

\title{
Dessin inédit d'une mosaïque de Vienne (Isère)
}

\author{
Henri Lavagne
}

\section{To cite this version:}

Henri Lavagne. Dessin inédit d'une mosaïque de Vienne (Isère). Gallia - Fouilles et monuments archéologiques en France métropolitaine, 1979, 37 (1), pp.101-117. 10.3406/galia.1979.1597 . hal01935514

\section{HAL Id: hal-01935514 https://hal.science/hal-01935514}

Submitted on 3 Mar 2020

HAL is a multi-disciplinary open access archive for the deposit and dissemination of scientific research documents, whether they are published or not. The documents may come from teaching and research institutions in France or abroad, or from public or private research centers.
L'archive ouverte pluridisciplinaire HAL, est destinée au dépôt et à la diffusion de documents scientifiques de niveau recherche, publiés ou non, émanant des établissements d'enseignement et de recherche français ou étrangers, des laboratoires publics ou privés.

\section{(이) $\$$}

Distributed under a Creative Commons Attribution - NonCommercial - NoDerivatives 44.0 


\title{
DESSIN INÉDIT D'UNE MOSAÏQUE DE VIENNE (Isère)
}

\author{
par Henri LAVAGNE
}

L'ourrage de F. Artaud ${ }^{1}$, Mistrire abrégée de la peinlure en mosaüque, suivie de la descriplion des mosäques de Lygon el du midi de la France, paru à Lyon en 1835), constitue pour toutes les études sur la mosiäque gallo-romaine un travail de référence d'une importance exceptionnelle. Les cinquante-huit planches aquarellées qui composent ce grand in-folio sont précédées d'une brève synthèse sur l'évolution de l'art de la mosaïque, et chaque planche est accompagnée d'une notice succinete qui relate les circonstances de la découverte et donne un bref commentaire sur le style du pavement examiné.

Mais on oublie souvent que ce texte élegant et dense s'appuie sur une recherche considérable, menée sur place par Artaud entre 1812 et 1835, et dont nous arons le témoignage sous forme de liasses de documents, carnets de notes, croquis, etc. conservés aujourd'hui par les soins de l'Acallémie des Sciences, Belles-Lettres et Arts de Lyon². En dépouillant systématiquement ces manuserits préparatoires ${ }^{3}$, nous avons eu la chance de décourrir un dessin d'une mosiäque de Vienne, aujourd'hui détruite, qu'Artaud n'avait pas juge bon de mettre au net et de reprorluire dans son ouvragre, et qui, pour cette raison avait jusqu'ici échappé à l'attention des spécialistes. Ce dessin se présente sous l'aspect d'une simple ébauche, mais les annotations qui le complètent sont suffisamment précises pour qu'il soit possible de restituer la presque totalité des parties manquantes, à l'exception des motifs figurés". Outre qu'il a l'intérêt de nous montrer comment Artaud lui-même. exécutait ses relevés, ce document nous fait connaitre une mosaïque sur laquelle nous n'avions aucun renseignement.

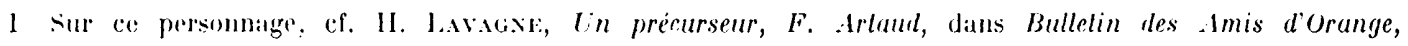
$11^{\circ} 68,1977$, p. $8-10$.

? Nous tenons a remercier M. Wasner, Conservateur do la bibliothégue de l'Académie, et M. Hours, Conserviteur des Archives municipales, pour l'amahilite avec lacquelle ils nous ont repu. Nous remercions également M. R. Lanxerois, Conservateur des Museres de Vieme, dont lade sur le lerrain et dans les archives de sainte-Colombeles-Viemne, nous a ete d'un grand secours.

3 Manuscrit no 101 (289), lome 1.

1 Cous devons celte restitution à lichard prudhomme, sans qui cette étude naturail pas été possible.

5 Xous ne traiterons pas ici de ce sujet. Il suffit de dire que chaque détail est noté avec minutie 'nombre 


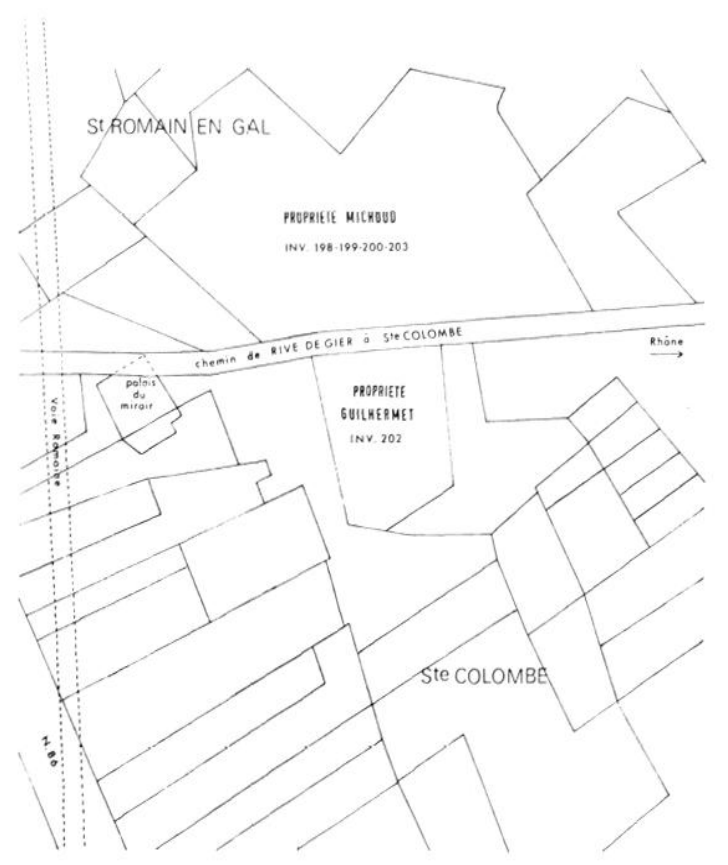

1 Vieme. Quartier de Sainte-Colombe, d'apres to carlastre de 1808.

L.'Ineenlaire des Mosä̈ques de la Gaule dù à G. Lalinge se contente de reprendre deux lignes de J. Savigné qui. dans son IVistoire de Sainle-Colomber, indiquait qu'une mosiäque "denviron lis metres sur 7 " avait eté découverte en 18\%2, "au rlos de II. Guilhermel, appelé la Charite, an midi des ruines du Palais du Viroir ». La description, tres médiocre. "ronds et carrés entoures d'entrelars ${ }^{8}$ permettait seulement de deviner qu'il devait s'agir d'un pavement dit "à décor multiple ", où médaillons circulaires et panneaux carrés etaient disposés en alternance dans une trame en quadrillage. La note d'Artaud n'est guère plus explicite quant a la localisation de la mosiäque : "chez 11. Guilhermet, à qui appartient la Tour du Rhòne, an 1822), derrière M. Michoud ». Mais il est possible, en étudiant le releve cadastral de 1808 et le plan de la commune de SainteColombe dressé à la mème époque", de préciser l'emplacement du pavement.

Lat famille Guilhermet blait propriétaire de plusirurs pareelles sur le territoire de la commune, entre le Rhòne au nord. le "chemin de Rive-de-(iere à Sainte-Colombe". et lactuelle route nationale 86 (fig. 1). Matis la sente terre appartenant à un Guilhermet ${ }^{10}$

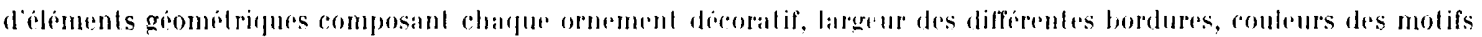

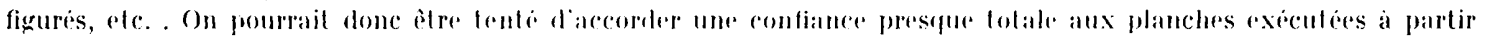

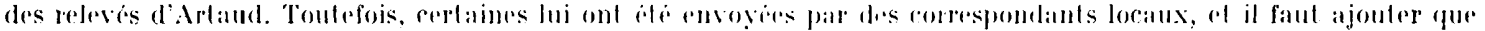

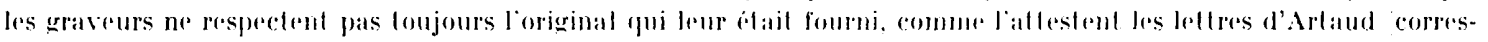
pondance indide conservere alux Archives municipales dolonge.

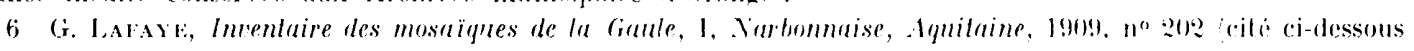
Inv. Mos. I .

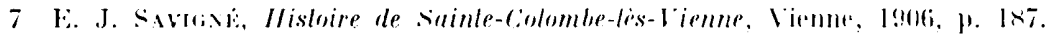

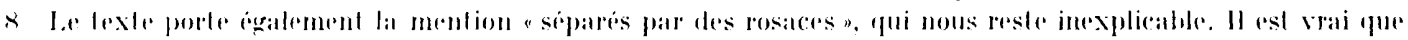

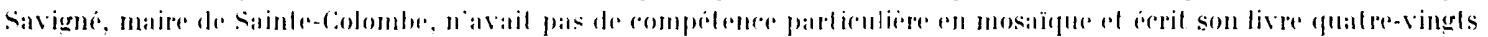
ans apres la décomiremle.

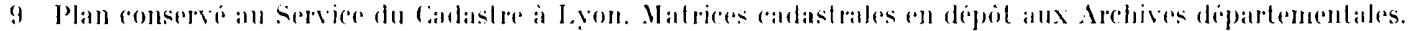

10 "Fleury Guilhermet, anbergiste". 


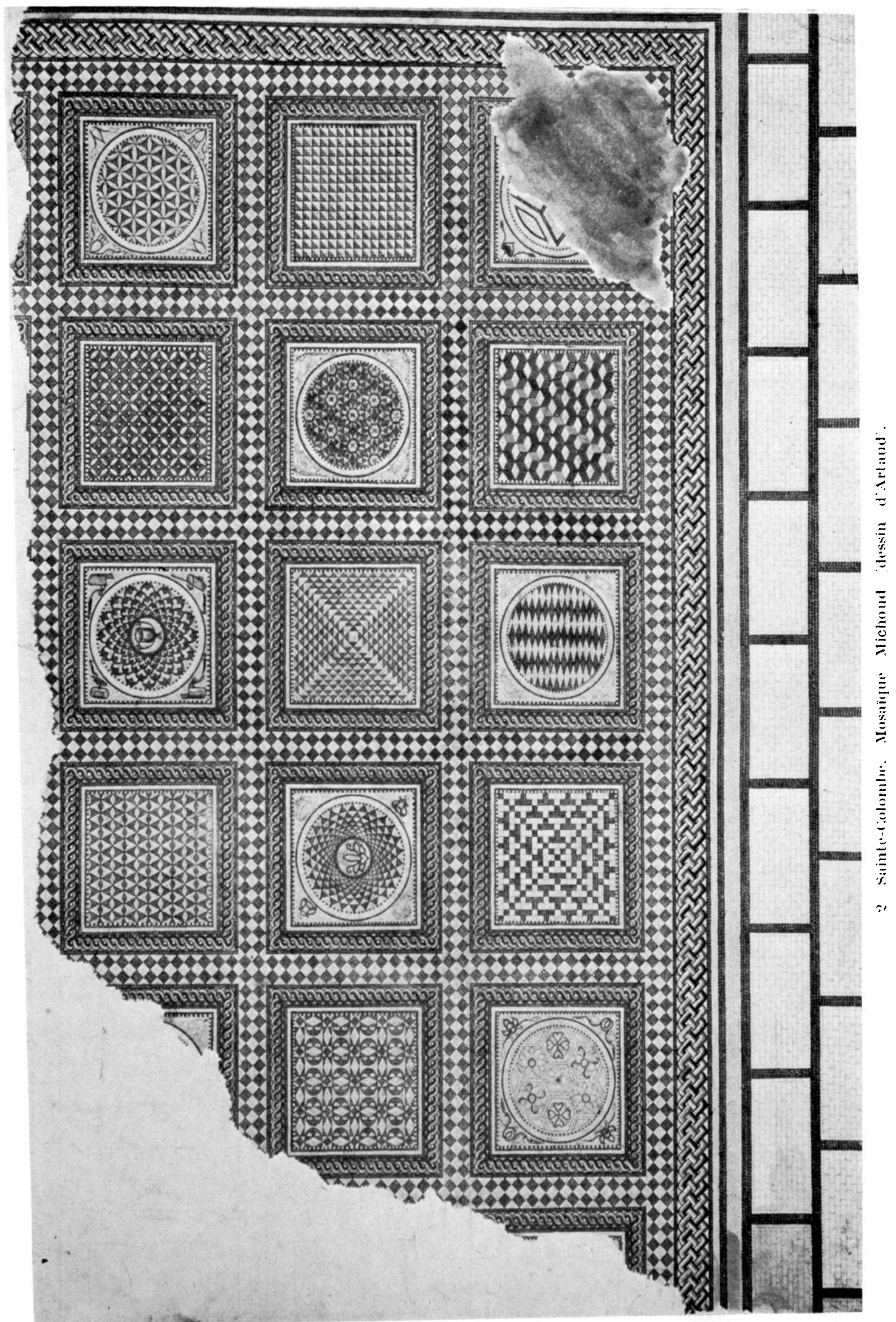




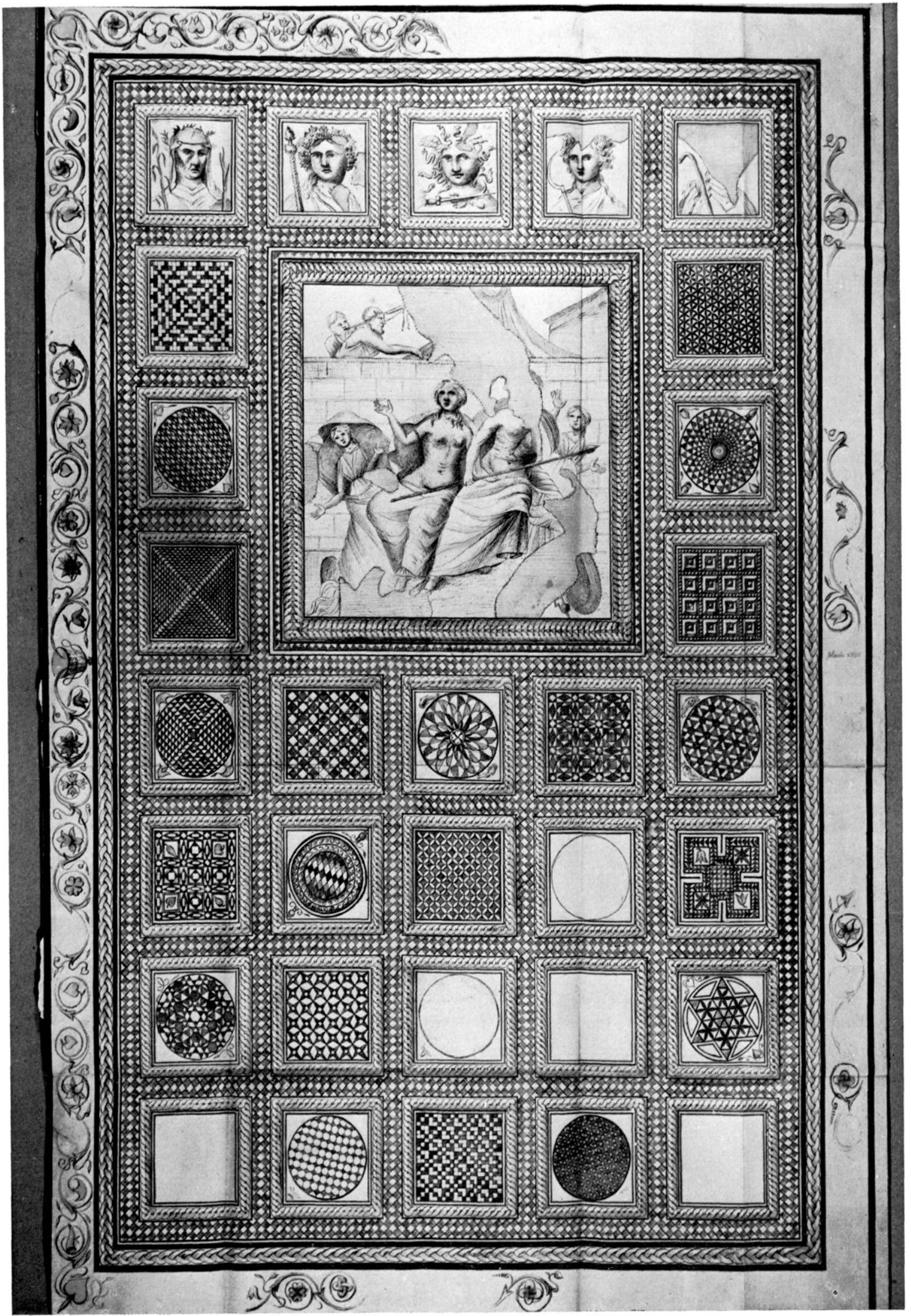

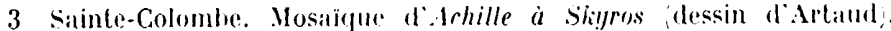


qui soit à la fois "au midi des ruines du Palais du Miroir" et "derrière le terrain de .I. Michoud" est celle qui porte le no cadastral 143. Elle est située à la limite des deux communes de Sainte-Colombe et de Saint-Romain-en-Gal, et Artaud devait nécessairement la connaître puisqu’il était lié avec Michoud qui l'avait autorisé à pratiquer des fouilles dans son terrain en $1811^{11}$. Toute la zone limitrophe du Palais du Miroirr2 fut d'ailleurs extrêmement fertile en découvertes archéologiques pendant le xix siècle : "torses, pieds, mains et têtes s'y sont rencontrés par charretées "écrit Artaud ${ }^{13}$.

On peut penser quion est en présence du prolongement du quarlier résidenticl de Saint-Romain-en-fial que les fouilles récentes ont mis au jour. La mosaïque Guilhermet aurait done orné quelque riche villa suburbaine du faubourg de la rive droite du Rhône. Mais la localisation exacte de cette mosaïque n'a pas simplement un intérêt topographique; clle permet également de la situer à proximité immédiate d'autres pavements qui présentent avec elle des analogies de décor parliculièrement frappantes. Ce sont d'une part la mosaïque

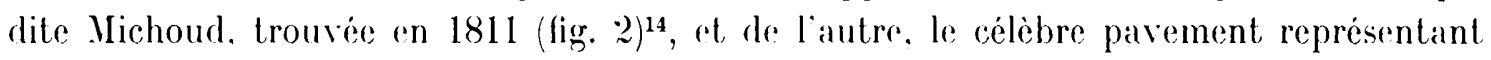
Achille à Skygros (fig. 3). dont Artaud nous dit qu il fut dégagé "dans la même vigne" que la mosaïque des Planètes ${ }^{15}$. Or, le plan de situation qu'il fournit ${ }^{16}$ montre que cette dernière était placée dans la mème domus que la mosaïque Vichoud. Il est impossible d'assurer avec une certitude absolue que la mosaïque Guilhermet faisait partie du même ensemble architectural que la mosaïque Michoud et celle d'Achille à Sliygros, mais il est hors de doute qu'elles sont l'œuvre d'un même atelier, comme le montre l'étude comparée de leur répertoire décoratif.

Les indications portées par Artaud au bas de son dessin, et les dimensions qu'il signale, autorisent à restituer un pavement d'une largeur de 6,40 m. La longueur est plus difficile à déterminer puisqu'il précise : «découvert sur 22 pieds (soit 7,04 m), le reste étant brisé. Il est à présumer que si on cherchait davantage on trouverait peut-être le tableau». L'hypothèse d'un "tableau ", ou pseudo-emblema. comme par exemple dans la mosaïque d'Achille à Skiyros, quoique probable dans ce genre de pavements à Vienne, n'est quiune hypothèse. Si l'on adopte le chiffre de 15 m fourni par Savigné pour la longueur, on a les dimensions d'une pièce considérable, arec un parement qui dépasse les plus grandes surfaces de mosaïques viennoises, puisque la mosaïque d'Achille à Skyres devait avoir $10,88 \mathrm{~m} \times 7,68 \mathrm{~m}$, et l'Ilercule ivre $10,33 \mathrm{~m} \times 6 \mathrm{~m}^{17}$. La fonction de la pièce qu'il décorait (triclinium?) reste également du domaine des suppositions.

11 l. Artaud, Hisloire abregee..., p. 80.

12 Sur cot édifice et les fouilles qui y furent pratiquées, cf. J. L.sBlaxc, Le Pronl du Rhône entre Vienne el

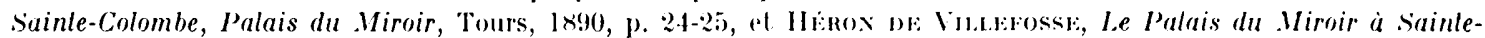
Colombe-lez-Vienne, dans Compte rendus Acad. Inscriptions et Belles-Lellres, 1907, p. 60.

13 F. ARTALD, Lyon souterrain, Lyon, 1834, p. 10.4.

14 Inv. Vos. I, $\mathrm{n}^{0} 200$.

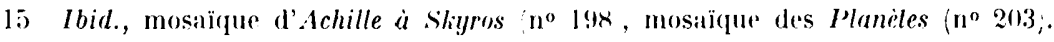

16 Artals, op. cil., p. 62, 79, 81 al plan de siluation, pl. Xl.

17 Hercule ivre, Ine. Mos. I, no 17.4. On ne connaît pas encore exactement les dimensions de da mosä̆que de Neptune de Besancon. Dans la premiere note publiese sur ce pavement, J.-P. Morel Informations archeologiques, dans Gallia, 32, 197.4, p. 404j donne comme mesures $11 \mathrm{~m} \times 16 \mathrm{~m}$, "si l'on suppose, comme il est vraisemblathe, que l'emblema... occupe te milieu de la composition ". colte hypothese est tres fragile, car dans la plupart des cas connus l'emblema est, an contraire, excentré. 


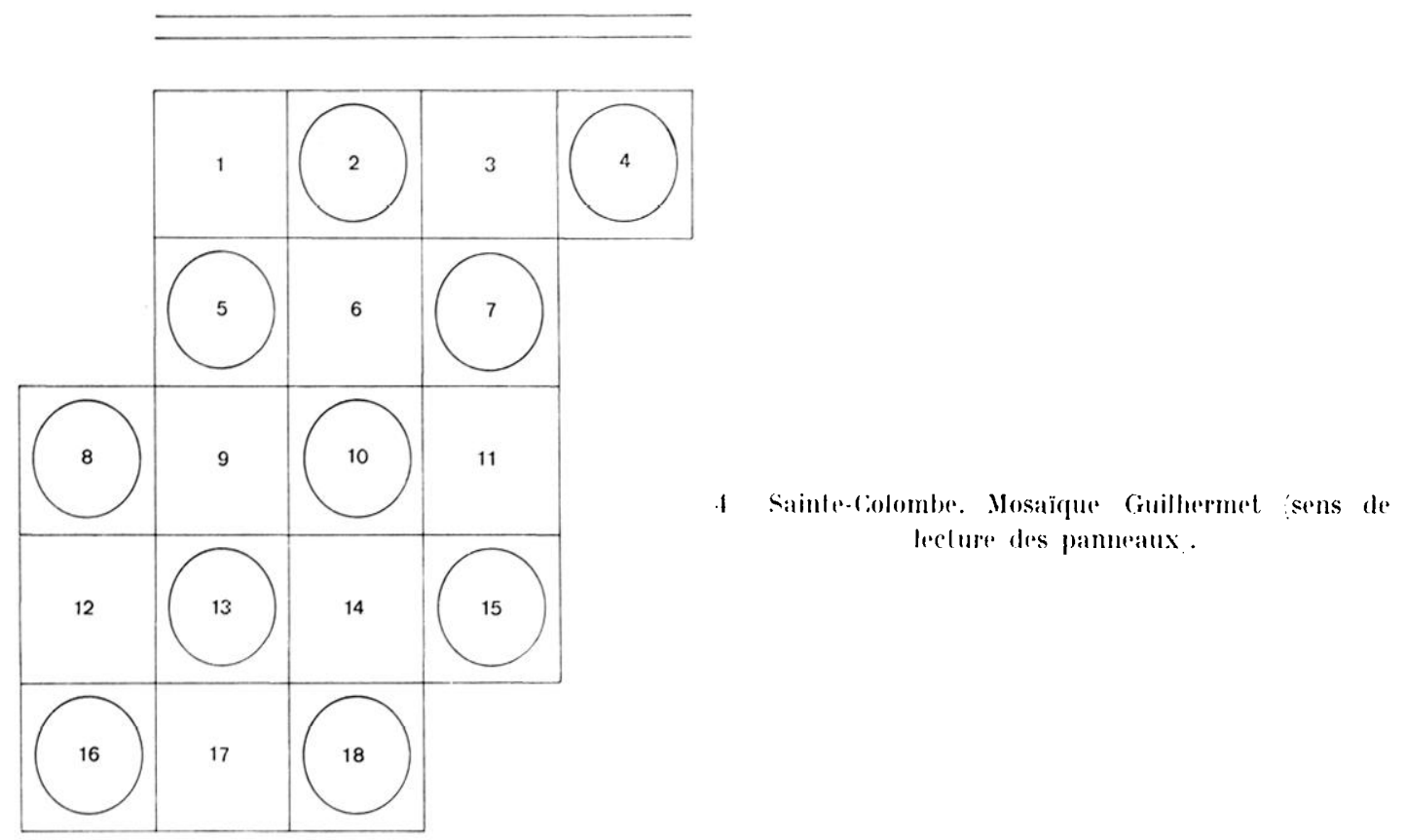

La composition du tapis n'a rien que de très classique : quarlrillage droit avee suecession alternée de médaillons circulaires portant dans les écoincons des motifs animés ou végétaux, et de panneaux carrés renfermant un décor géométrique couvrant la totalité de la surface du caisson (fig. 4 et 5). Chaque panneau $(1,06 \mathrm{~m})$ est entouré d'une tresse polychrome (larg. $10,8 \mathrm{~cm}$ ) à deux brins, l'ensemble étant relié par une double ligne do petits carrés noirs se touchant par les pointes.

Tous les motifs sont dessinés par un double filet de cubes noirs, comme c'est toujours le cas à Vienne ${ }^{18}$. Seule la bordure innove par rapport aux exemples connus jusqu'ici de cette trame. I)ans la mosaïque d'Achille à Skyros, on avait un rinceau véegétal relativement simple, dans la mosaïque Vichoud une tresse à quatre brins, dans la mosaïque dite Séguin (fig. (6). également trouvée à Sainte-Colombe ${ }^{19}$ un rare motif d'enroulement végétalisé et un guillochis: ici, la variante consiste à utiliser le méandre de svastika, accompanné d'une large bande de raccord noire $(10 \mathrm{~cm})$ et d'une bordure intérieure de trois rangs de cubes igalement noirs. Ajoutons une dernière notation d'Artaud, sur la technique : "Les cubes sont très fins ». L'état de conservation au moment de la découverte était médiocre, puisque sur les vingt-cinq cases du quadrillage dont l'existence est assurée par le dessin, dix-sept seulement etaient encore visibles au moment du relevé d'Artaurl.

Case $n^{0} 1$ : décor couvrant de sept ranogs d'octogones adjacents, noirs, chargés d'un rectangle blanc alternativement couché et dressé.

Case $n^{0} 2$ : roue de huit carrés sur la pointe, orné chacun d'un double carré emboîté inversé, en opposition de couleurs, ces carrés étant disposés entre les huit pointes d'une étoile blanche,

Ix Janine Lavina, Mosäques geomélriques. Les aleliers de Vienne (Isire), Rome, 1977, p. 2.4.

19) In'. Wos. I, no 15s. 


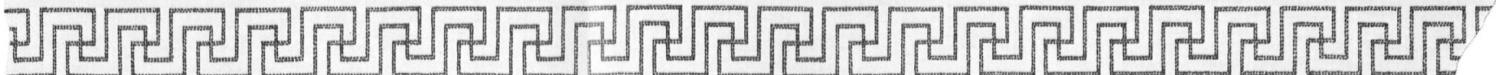

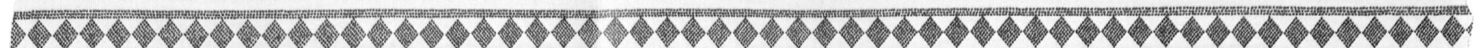

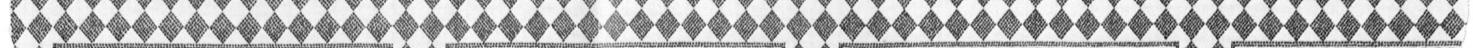

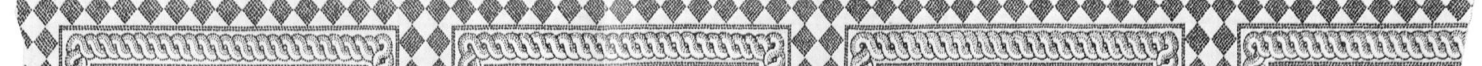
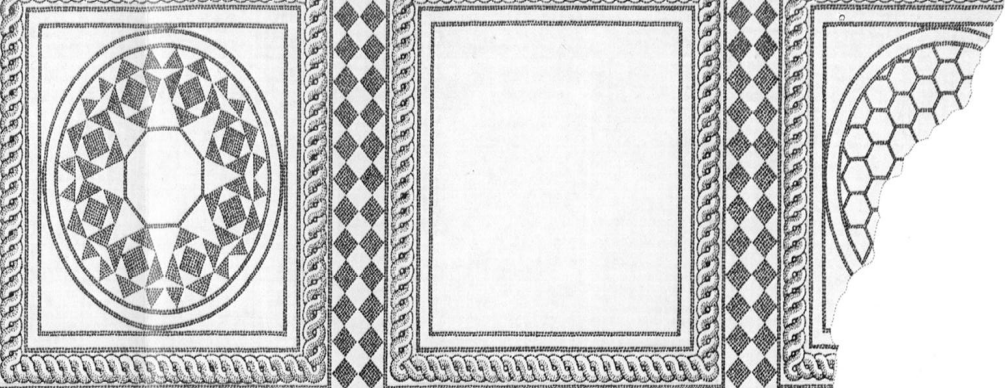

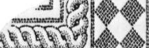

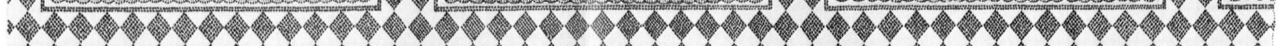

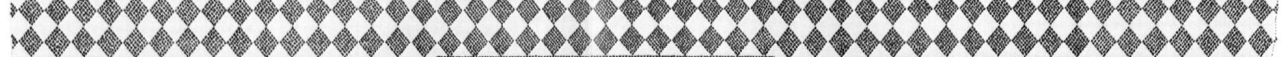
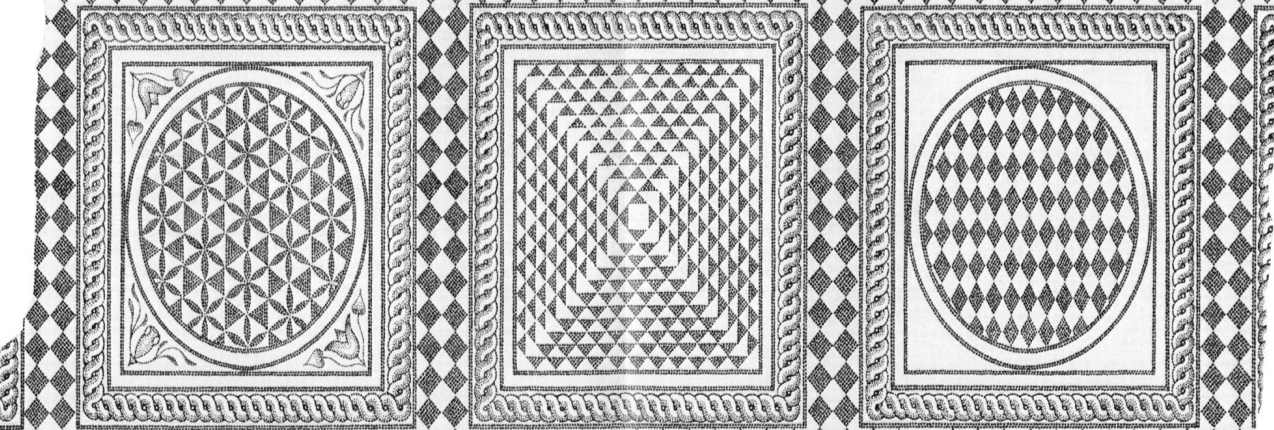

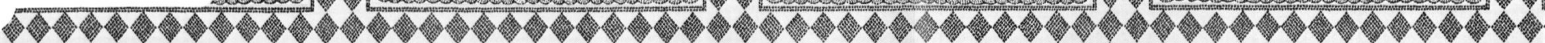

-
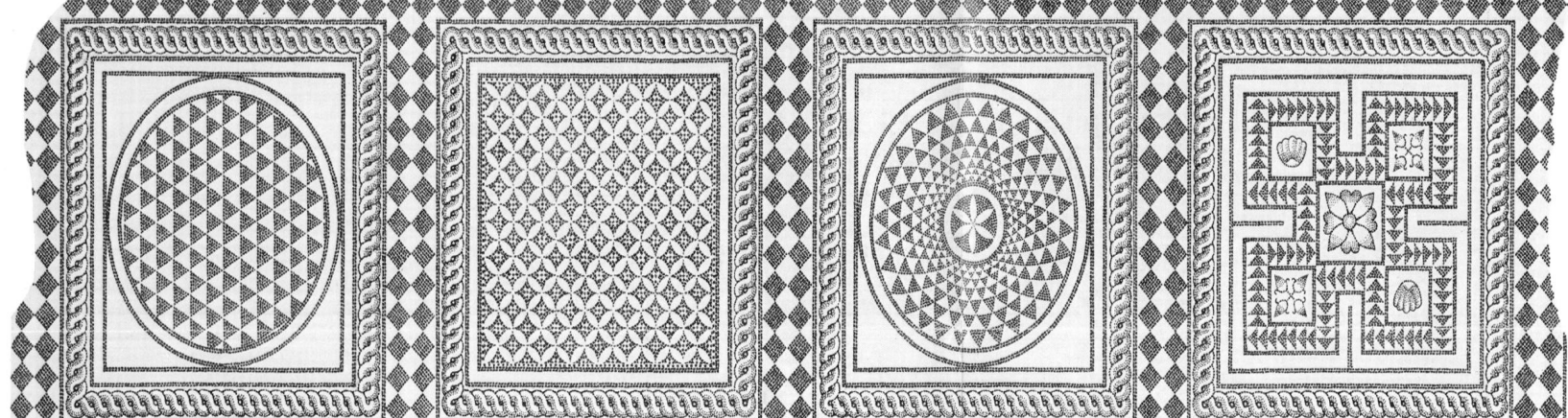

(c)

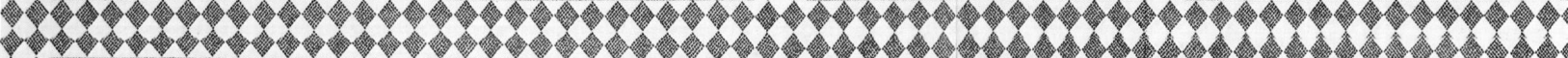

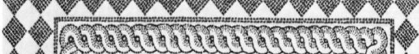

\section{8}
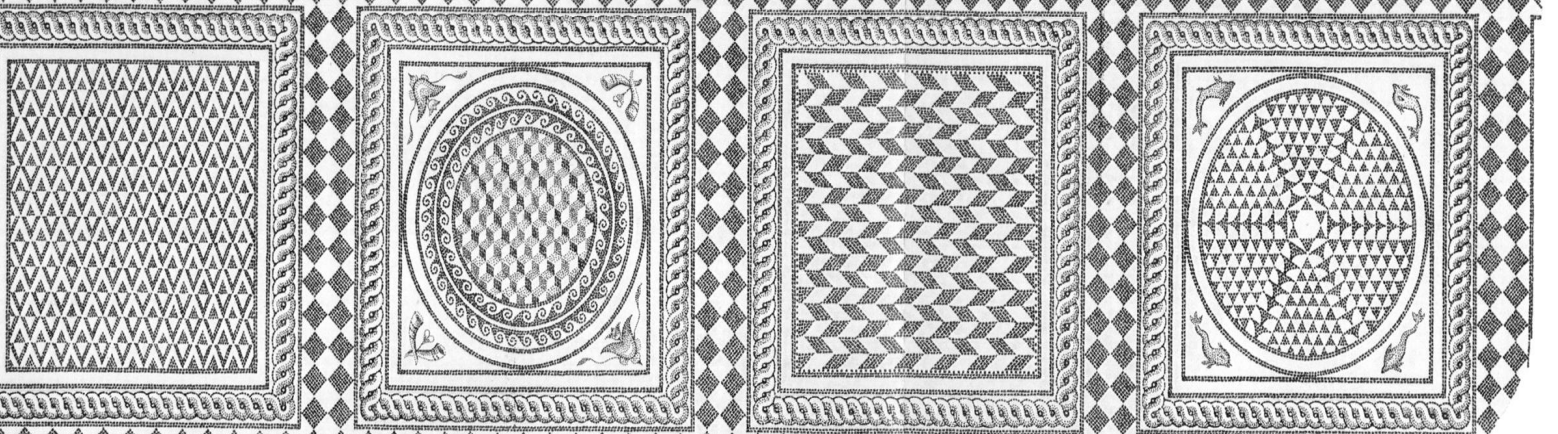

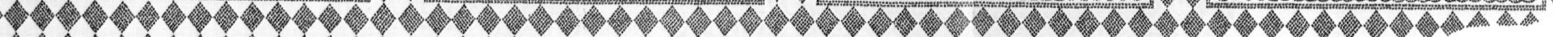

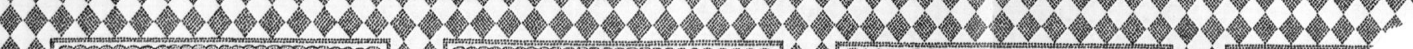
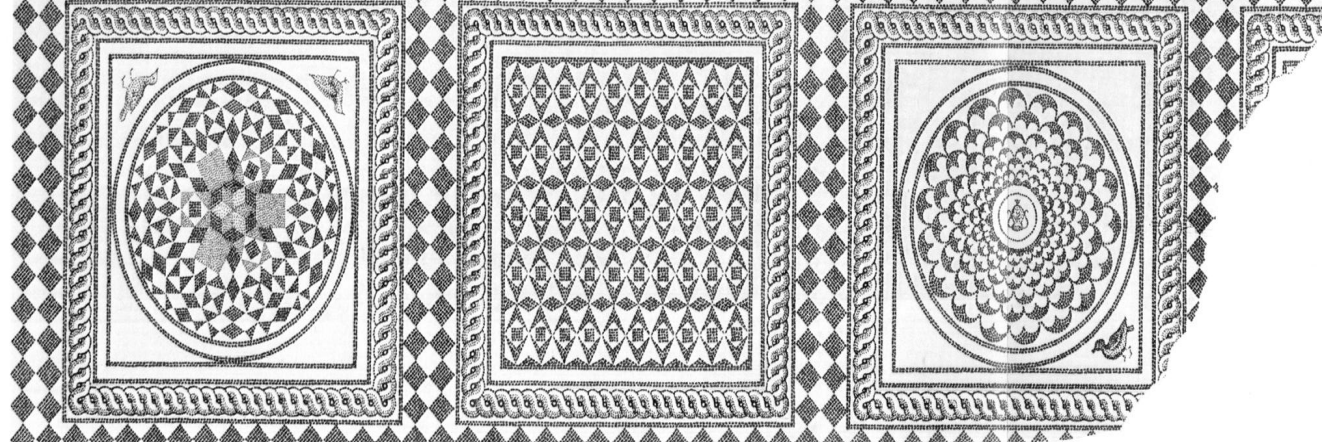

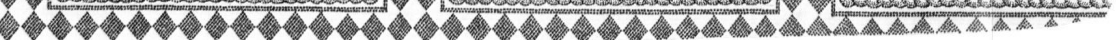


elle-mème frappée d'un octogone. Celui-ci devait comporter un remplissage interne, probablement un fleuron qu'Artaurl n’a pas dessiné. Ce motif radial apparait déja à Pompéi sous une forme approchante ${ }^{20}$, et revient a plusieurs reprises dans les pavements a décor multiple, mais presque toujours avec un aspect géométrique plus complexe. Ainsi. dans le fle caisson de la mosaïque de l'Iuresse d'Hercule (fig. 7 ; ou, entre les branches de léloile, sintercalent des losancres. Jous retrouvons ce jeu de carrés et de losanges dans la case 16, mais beauroup plus élaboré.

Case no :3 : probablemenl déja détruite au moment où Arlatud fit son relevé, car le dessin ne comporte aucune indication sur ce point.

Case no 4 : en partie "ruinée". note Artaud. Composition d'hexagones en nids d'abeille, chacun étant dessiné par un filet double de cubes noirs. Motif Irop fréquent pour qu'on y insiste, car il est employé ausisi bien comme déror de fond à loute époque. que comme remplissage de médaillons circulaires dans ce lype de mosaïques. On serait d'abord tenlé de penser qu'Artaud a oublié de préciser la façon dont blat garni chaque hexagone six-feuilles comme dans un pavement à décor multiple de lyon ${ }^{21}$ ou comme dans le f10e caisson de la mosäque de l'Irresse d'Ilercule?), mais il arrive également que ce molif garde sa simplicité linéaire, mème au milieu de caiscons très travaillés. comme c'est le cas pour le efe carré de la mosiäque d'Hercule.

Case $n^{0}$ ) : composition dhexagones langents par tous leurs sommets, determinant des lriangles équilatéraux. Les hexagones sont rharges d'un six-feuilles inscrit. en opposition de couleurs. Motif lui aussi tris fréquent et bien représenté a l’époque ancienne en Italie, puis, dés l'époque flavienne a Besançon ${ }^{22}$ et Orange ${ }^{23}$, le plus souvent avec le six-feuilles inscrit. Par sa structure, il se prête bien à garnir des médaillons rirculaires comme dans une mosilique de Vaison-la-Romaine ${ }^{24}$ ou dans un caisson de la mosal̈que d'Ldmète a Ximes où les hexagones sont timbrés d'un svastika.

Jans les écoinçons, deux motifs typiques des pavements a décor multiple sont notés par Irlaud : une amphore "rouge, jaune el blanche », entourée de deux tiges végétales a longues feuilles, et un calice de fleur "vert et bleu clair", encadré par deux feuilles cordiformes "rouges, jaunes et blanches". L'amphore apparaît, exaclement identique, dans le ge caisson de la mosaïque d'Achille a Sliyros; elle est accompannée de feuilles plus développées, avec des vrilles, dans le 1 je $^{\mathrm{e}}$ caisson de la mosäque séguin; dans le 42 e casson de lllercule inre, les vrilles naissent non pas du pied de l'amphore, mais des anses, comme dans le caisson décoré d'un chien courant du pavement no 187 de l'Inventaire de Lafaye ${ }^{26}$. Mais la ressemblance la plus proche est a chercher dans le dessin de l'amphore représentée dans la mosiäque Vichoud (je caisson) où les tiges se prolongent, comme ici, par un groupe de trois feuilles élancées. Cette identilé de faclure dans un détail mineur d'un motif passe-partout est pour nous un indice d'appartenance à un mème atelier. Le motif des calices et lleurs est également bien ronnu dans le décor multiple. mème si sa forme plus ou moins végétalisée peut varier aver chaque mosaïste. (On ritera seulement l'exemple du 20e caisson de la mosaïque

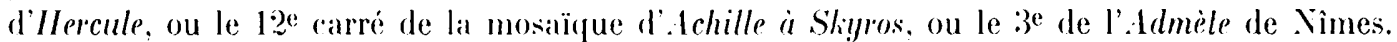

Case $n^{0}$; : composition de triangles rectangles adjacents, en opposition de couleurs, qui rayonnent en quatre directions, faisant apparaitre un molif en croix de siant-indré constituée par quatre bandes de triangles superposés. C'est une des rompositions les plus classiques du décor

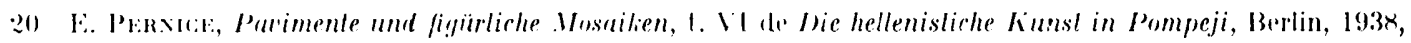
pl. . (I, il, i).

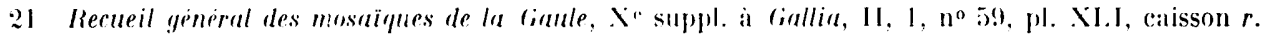

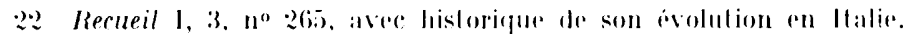

23 Rerueil III, 1, n" $5 \%$.

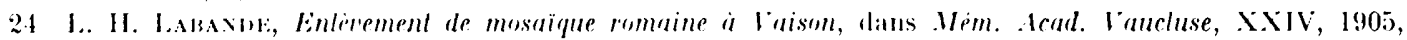

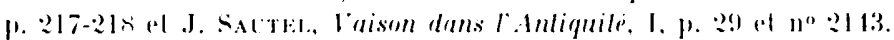

25) Inv. Mos. I, $\mathrm{n}^{\circ} 3299$.

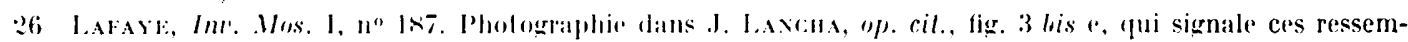
Hances, p. :21. 
multiple, héritée de motif's ilaliques ${ }^{27}$. Nous la trouvons par exemple dans le 17 e caision de l'Ilercule iore (la couleur des triangles est inversée : noirs sur fond blanc: mais aussi dans le loe carré de

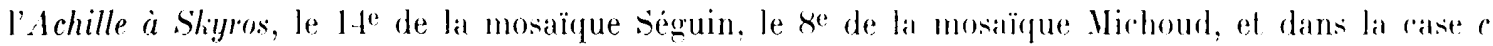
du pavement no 5.j) de Lyon, ou $j$ du no j9 de la mime ville.

Case no 7 : damier de losanges en opposition de couleurs. Composition géométrique lress simple, qui peut exister également en opposilion de trois couleurs comme dans le l fe caisson de l'alchille a Sligros, mais ligure, identique, dans le 1:3e carré de la mosaïque Vichoud, el dans la case y de la mosaïque $n^{0}$ :) de Lyon.

Case $n^{\circ} 8$ : le médaillon circulaire est entierement rempli par une composition en damier de triangles équilatéraux, en opposition de couleurs. Le desin nötant pas arhevé sur le document laissé par Artaud, il est possible que le centre du motif ait eti un hexagone comme dans le 3ye caisson de la mosaïque de l'Hercule irre.

Case $n^{\circ} 9$ : composition de cercles sécants déterminant des quatre-feuilles blancs et des rarrés curvilignes noirs charges d'une croiselle. Motif extremement courant aussi bien en tapis de fond que dans le décor multiple. On comparera avec le 1 ge caison de l' Lehille à Sligros, ou avec le 4e carré de la mosäque Michoud. On soulignera ici que le filel intrieur qui forme le carlre du rarré est a denticules noirs et blanes, comme dans la case no 14.

Gase $n^{\circ} 10$ : bouclier de triangles frappé en son centre par un six-feuilles blanc sur fond noir. Thème décoratif des plus fréquents, qui revient particulierement souvent dans le décor multiple, notamment dans le ge caisson de la mosaïque Vichoud faver un fleuron au centrej, dans le ge de

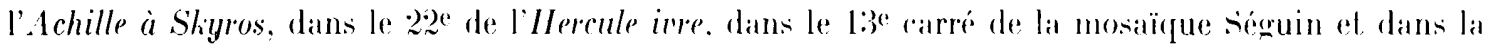
case g de la mosaïque $n^{0}$ 5.3 de Lyon.

Case no 11 : le décor de cetle case est plus original el reprend, en réduction. une structure dite

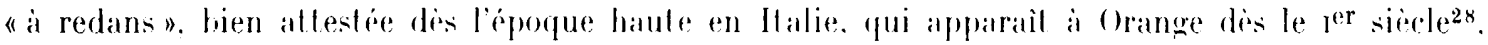
et connaît son plus grand développement en Gaule et en suisie a partir de la seconde moitié du ${ }_{1}{ }^{e}$ siecle ap. J.-C. Il semble que la bande de triangles superposis qui souligne les ressials soil le traitement le plus ancien par rapport a la lresie. Le schema délermine quatre carrés en encoignures et un carre eentral. Celui-ci est charé d'un petit fleuron de type rhodanien courant fqualre pritales lancéolés en croix entre quatre pétales cordiformes dont la vive polychromie est notée par Irtaud: "La lleur du milieu esl rouge, blanrhe, el jaune. les corurs verts el bleus. " lans les quatre carrés d'angle, coquille alternant aver un fleuron romposite formé de qualre calices pointus. Ie schéma a redans dans le décor multiple nest pas inconnu, mais il est souvent plus élaboré : dans le ge caisson de l'Hercule ivre. il délermine huit ressauts, aver un traitement analogue en lignes de triangles: superposés, mais dans le 27 e carré, il est rendu par une lresse, aver dans les deux ras, des remplissages purement géométriques. Ies coquilles ici représentés sont présentes dans les 22e et 32e raissons de l'Hercule iore, dans le 10 e carré de la mosaïque séguin qquoique beaucoup plus développées el dans le 28 e de l'Achille à Sligross.

Catse no 12 : composition courante de raissons bipartiles losangés en opposilion de couleurs. Sous cette forme, le molif n'apparaît dans aucun aul re exemple de décor multiple.

Case no 13 : probablement le medailon le plus soigné de loute la mosiäque. On remarque qu'il est le seul a comporter une double bordure interne. constiluée par deux lignes de postes opposies: de part et d'autre d'un filet blanc. Le centre du médaillon est couvert par une composition de cubes adjacents d'une belle polychromie (rouge, vert, jaune, relice Artaud), avec effet de trompe-l'aril.

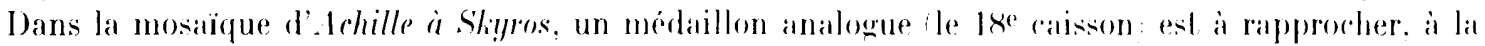

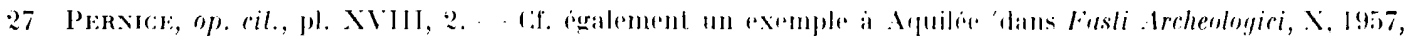
p. 342, no 4292, fig. 110 at it Altino dans . Vol. tical., 1930, p. 46.4, fig. 1.

2x Voir l'origine al l'exolution du schena dans Recupil III, 1, no 3x. 
fois par sa double bordure de postes, par son décor tris apparenté (le damier de losanges et une polychromie également recherchée. I)ans les deux mosiliques, il occupe une position à part, au milieu de médaillons el de panneaux carrés qui ne comportent aucune bordure intérieure ${ }^{29}$. Ce trail peut également indiquer une origine commune aux deux pavements. Le $19^{\mathrm{e}}$ et le $34^{\mathrm{e}}$ caissons de la mosä̈que d'llercule irre. quoique légrìrement différents, sont traités dans le mème esprit. Dans les écoinçons, les remplissages figurés appartiennent au répertoire traditionnel du décor multiple : les doubles rhytons sont identiques a ceux de la mosä̈que séguin (15) caisson), mais apparaissaient aussi dans le 12 e caisson de la mosaïque d'Hercule (avant sa restauration), à l'état simple, comme dans le fragment $\mathrm{F} 1$ du pavement $\mathrm{n}^{0} 213$ de l'Incentaire ${ }^{30}$. Les deux autres écoinçons sont remplis par des ralices a deux corolles, arcompannées de deux feuilles allongées, l'ensemble étant polychrome.

Casse $n^{0} 14$ : damier de losanges blanes et noirs, traité en chevrons décalés, ici souligné a l'intérieur du cadre du carré par une ligne denticulée comme dans le caisson 9. Il est intéressant de relever que ce détail est commun arec les quinze panneaux de la mosaïque Michoud et avec les $12^{\mathrm{e}}$ et $14^{\mathrm{e}}$ caissons de la mosiäque séóguin, alors que cette particularité est absente de loutes les autres mosaĭques a décor multiple de la vallée du Rhòne. On la retrouve toutefois dans trois panneaux de la mosaïque d'Ouzouer-sur-Trézée ${ }^{31}$. Ce trail est ancien, fréquemment attesté à Pompéi ${ }^{32}$ et dans les mosaïques du $1^{\mathrm{er}}$ siecle de la Provence romaine, a Orange, Vaison, Apt ${ }^{33}$, elc. Le damier de losanges existe également dans la mosaïque $\mathrm{n}^{0} 53$ de L,yon (rase $c c$ \%.

Case $n^{\circ} 15$ : a partir d'un hexagone central chargé d'une étoile à six pointes, composition radiale de triangles équilatéraux rayonnant en six direclions. et séparés par des bandes d'épines noires sur fond blanc. Ce schéma est une variante de celui qui ligure a la case ${ }^{0} 6$. On le retrouve également dans le 12e caisson de la mosaïque d'. Ichille à Skyros. Dans les écoingons, quatre dauphins en positions opposées. dont Artaud donne les couleurs : « corps vert et bleu-clair, nageoires et queue rouges ». Ces dauphins, dont la forme sinueuse prète souvent à garnir des écoinçons, se retrouvent également dans le $16 \mathrm{e}$ caisson du parement d'.lchille et dans le $6 \mathrm{e}$ de l'IIercule iore, sans oublier le fragment $\mathrm{F} 2$ du no $21: 3$ de l'Intentaire ${ }^{34}$.

Case $\mathrm{n}^{0} 16$ : c'est la composition géométrique la plus complexe de tout le pavement. Au centre, une étoile de six losanges polychromes est inscrite dans un hexagone a fond noir, flanqué sur ses còtés de six carrés alternativement roses el blancs sur fond noir (carré emboîté inversé); entre ces carrés sont disposés six losanges quadripartites en opposition de couleurs. Sur ce motif s'appuie une seconde rangée composée de carrés sur la pointe, en ligne concentrique à la première. Enfin, sur les còtés des six premiers carrés sont placés six triangles équilatéraux qui complètent ainsi les pointes d'une étoile à douze branches dont les six autres sont les losinges quadripartites. Ce jeu trís savant sur l'hexagone, le carré et le losange étroitement imbriqués, est, en fait, la complication du motif de la case 2. Il est a comparer également avec les $10^{\mathrm{e}}$ et $44^{\mathrm{e}}$ caissons de la mosaïque d'Hercule ou avec le 22 e de la mosaïque d'Achille, ou encore avec la case $z$ de la mosaíque $n^{0} 53$ de Lyon. Mais dans ces trois derniers cas, la construction du motif est fondée sur les rectangles qui s'appuient sur les còtés de l'hexagone central, alors que dans notre mosaïque, le molif de base qui permet cet combinaisons est le carré, comme dans la case o de la mosaïque no 59 de Lyon. On peut également noter des compositions presque analogues dans le ge caisson de la mosaĭque Michoud, ou le $15^{\mathrm{e}}$ de

29 I.e fait est remarqui par J. I.ancha (op. cit., p. 2l.

31) I,Axina, op. cil., lig. $x$.

31 Recueil $11,3, n^{\circ} .167$, caissons $i, 0, r$.

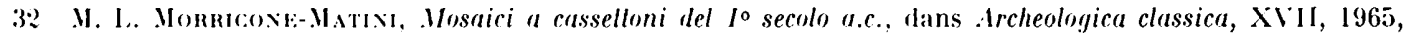
p. $\times 6$, n. 33 .

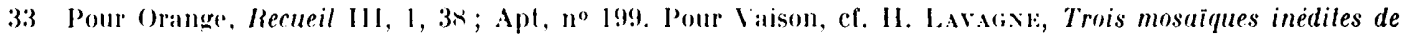
Vaison-la-Romaine el de Sainl-Paul-Trois-Chaleaur, dans Reve archéologique de Narbonnaise, . 1977, p. 172, fig. 1.

34 J.axcha, of. cil., fig. $x$, an has de la planche. 





la mosaïque séguin. lans les écoincons, le desin d'Artaud donne deux volatiles, du type caille ou perdrix, polychromes. Ce motif est tress répandu dans les éroinģons des mosaïques viennoises, particulierement dans l'Hercule ire caisons $\mathrm{n}^{\text {os }} 2,8,16,24$. 28, mais aussi dans le fragment $d$ du pavemenl $n^{0} 187$ de l'Inrenlaire ${ }^{35}$, el dans les panneaux a et $c$ du no $197^{36}$. I Jans la mosaïque Vlichoud, les oiseaux reparaissent également. mais un peu différents puisqüils picorent une branche.

Case $n^{0} 17$ : damier de losanges chargés alternativement d'un petit losange en position inversée, ou d'un carré sur la pointe blanc, inserivant lui-mème un carré noir droit. Composition très rare, qui

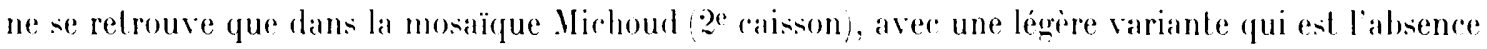
du carré emboilé en opposition de couleurs.

Case no 18 : composition de six lignes concentriques de peltes noires sur fond blanr. Lat derniere rangée avant le cercle central est une simple ligne de festons noirs. Dans un écoincon, un canard, le reste niayant pas été dessiné. Ie motif des pelles en rangs concentriques est assez rare, mème dans le décor multiple. On le renontre une fois dans la mosaïque Vlichoud be caisson avec le mème canthare au centre du cercle, mais aussi dans les fragments Fo et Fit de la mosaïque $n^{0} 213$ de l'Inrentaire ${ }^{37}$. C'est une variante plus slylisée dans laquelle les peltes ne sont que des écilles, que l'on peut relever dans un caisson d'une mosiäque de Lyon ${ }^{38}$, ou dans la mosiäque de teptune récemment découverle à Besancon ${ }^{39}$.

L'analyse détaillé de ces motifs, el le releve des comparaisons auxquelles alle contraine. si fastirlieux qu'il y paraisse, était néanmoins nécessaire pour préciser la place de ce pavement dans une série hien illustrée, particulièrement à Vienne et à Lyon. Cne partie du décor (rases 4, 5, 7, 8, 9, 19, 1:) appartient à un répertoire ornemental très rommunément utilise par toutes les mosaïques qui participent de ce que l'on appelle le "décor multiple". les autres motifs ('ases 2. 6, 11, 13, 15, 17, 18) sont plus originaux et ne se retrouvent que dans la mosiäque Michoud, la mosiäque Séguin et le pavement d'Achille a sliyros. Encore faut-il souligner que la proportion des traits communs arec la mosaïque Michoud est, nettement plus forte, dans la mesure où celle-ci ne comporte que quatorze cases conservees, contre vingt at une dans l'Achille chez Lyjcomède. Si l'on rappelle que la mosaïque Viehoud était située a proximité immédiate de la mosaïque Guilhermet, il parait légitime de considerer quedles sont toutes les deux les ceures d'un mème atelier travaillant peutetre pour le même proprietaire. Il nous semble simplement probable que le parement d'Achille à Skyjres doit être également attribué à cet atelier. On restera plus sceptique sur l'appartenance de la mosiäque séguin a cette mème série. son caractere tress hacunaire interdisant, all reste. des rapprochements suffisamment nombreux pour etre probants. Ces trois mosaïques viennoises ne sont malheureusement pas datées de facon sùre et leur confrontation avec la mosil̈que (iuilhermet ne peut done servir à situer - ipso facto - celle-ci dans le temps. On pourrait ètre tente d'avoir recours aux pavements trourés dans le Couvent du "Verbe Incarne " à Lyon" dont nous avons note certaines analogies aver: la mosä̈que qui nous ocerupe mais. la ancore. Jes datations sont contestées. Il nous faut

35) Hoid., fig. 3 bis, fragmmenl d.

36 Ihid., fig. 1.

37 Jbidl. ligr. $r$.

$3 x$ Recueil II. 1. n"

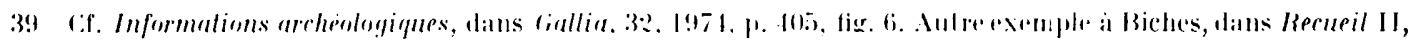

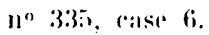

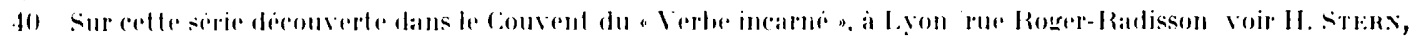

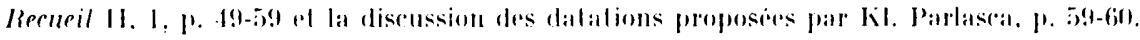


done reprendre l'examen de ce dossier pour essayer de préciser la place ... au moins dans une chronologie relative -... de la mosaïque Guilhermet.

Dans un article réunissant les principaux exemples du "décor multiple" dans la Vallée du Rhône, II. Stern ${ }^{41}$ a montré que ce répertoire était rommun à divers types de: trames, notamment all quadrillage droit (ou en échiquier), et à sa variante, le quarlrillage: de bandes croisées. Par la suite ${ }^{42}$, le même auteur a précisé la datation qu'il arait proposée (première moitié du nI $^{\mathrm{e}}$ siècle) en indiquant que la mosiäque de l'Hercule iure ct le parement en quadrillage de bandes croisées $n^{0} 173$ de l'Ineentaire. appartenaient au même atelier, travaillant "dans la deuxième et troisième décade du II $^{\mathrm{e}}$ siècle ", situant du mème coup les mosaïques de Lyon nos 53, 58, 59 ("Verbe Incarné») du Recueil à la même époque.

L'ouvrage récent de Janine Lancha ${ }^{43}$ remet en cause cette datation traditionnellement admise et propose une évolution chronologique dans laquelle les deux séries de trames se succéderaient. Dans une première phase (première moitie du II $^{\mathrm{e}}$ siècle) apparaîtraient les quadrillages droits, puis, entre 150 et 220 , se développerait une deuxieme phase au cours de laquelle le quadrillage de bandes croisées remplacerait le quadrillage droit. en lui empruntant des éléments de son répertoire décoratif. Selon ylue lancha, trois parements pourraient illustrer ces différents tournants : la mosiäque de l'Ilercule ivre serait à situer dans le troisième quart du II $^{\mathrm{e}}$ siècle et constituerait "le chef-d'œure du décor multiple" dans une grille en quadrillage droit; le no 173 de l'Intenlaire aurait été "posé sans doute en même temps ou peu après ", et serait "la première ébauche du quadrillage de bandes croisées inspiré des réussites du canevas à décor multiple "44. Dans "le dernier quart du II $^{\mathrm{e}}$ siècle ou au début du ni ${ }^{\mathrm{e}}$ siècle », on placerait le $\mathrm{n}^{0} 190$ de l'Invenlaire (aujourd'hui au Musée Saint-Pierre de Vienne), qui représenterait "la forme la plus achevée du quadrillage de bandes croisées ". La mosaïque d'Anse (Rhône) ${ }^{45}$ ferait la charnière entre ces deux phases puisquion y voit un "asscmblage de ces deux canevas". Il faudrait la dater du "troisième quart du $\mathrm{II}^{\mathrm{e}}$ siècle ". La séquence ainsi proposée retient de lanalyse d'H. Stern, que l'Ilercule iere ( $\left.\mathrm{n}^{\circ} 174\right)$ et le $\mathrm{n}^{0} 173$ de l'Invenlaire appartiennent bien au mème atelier, mais elle les remonte tous les deux au troisième quart du zie siècle. Cé décalage n'est pas sans importance puisqu’il entraîne à sa suite les mosaïques de Lyon construites sur les mêmes canevas; et notamment la plus grande des mosaïques du "Verbe incarné "4 qui passe du premier quart du II $^{\mathrm{e}}$ siècle (selon H. Stern) au troisième quart du $\mathrm{I}^{\mathrm{e}}$ siècle pour J. Lancha.

Le point d'ancrage de cette nouvelle chronologie est évidemment la date des nos 173 et 174 de Vienne. Si l'on accepte l'hypothèse d'A. Pelletier dans sa thèse sur Vienne, qui indique que ces deux parements faisaient partic d'une même domus détruite à la fin

41 II. Sтвк, deliers de mosäsles rhodaniens déporque gullo-romaine, dans Colloque de la mosaïque grecoromaine, Paris, 1963, p. 23:3-24:4.

42 Iо., Deux mosä̈ques de Vienne, dans Monuments P'iol, 56, 1965, p. 1:2.

13 (if. n. 1 .

44 Laxcha, ibid., p. 30.

45 Pour Anse, Recueil II, 3, no 175.

46 Recueil 11, 1, no 53.

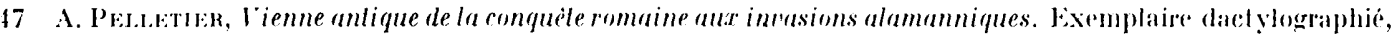
I, p. 3206 . 


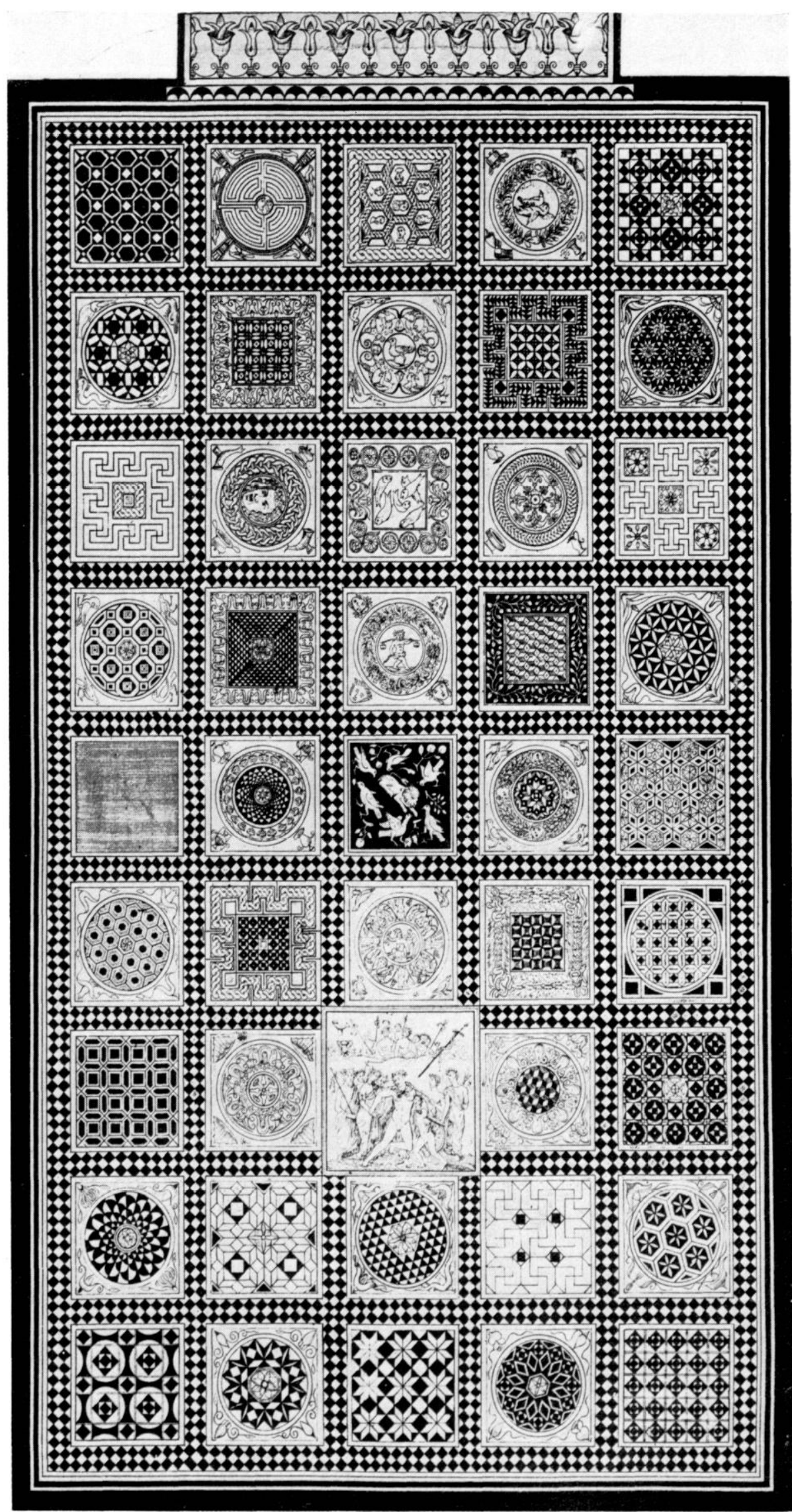

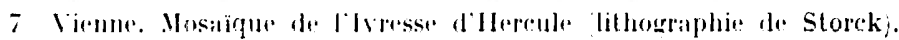


du II $^{\mathrm{e}}$ siècle, et que le quartier où elle était située sur la rive gauche du Rhòne, une fois inondé par une crue catastrophique, ne fut pas récecupé par la suite, nous tenons là un lerminus de première importance : en tout etal de cause, les $11^{\text {os }} 173$ et 174 de Vienne ne sauraient plus ètre placés au $\mathrm{II}^{\mathrm{e}}$ siècle, comme le voulait H. Stern.

On pourrait étayer cette affirmation en soulignant qu'à L,yon, on peut également s'appuyer sur un terminus acceptable, pour trois des mosaïques du "Verbe Incarné": d'une part, la présence d'un diplòme militaire trouvé sur le pavement en quardrillage de bandes croisées no 53 , et daté du 16 mars 192, de l'autre, l'incendie de Lyon en 197 lors de la victoire de Septime-Sévère sur Albin. Cet événement est précisément rattaché à l'histoire de ces mosaïques par Kl. Parlasca48 qui veut dater les réparations apportées à la mosaïque no 61 des lendemains de la victoire sur Albin. Or, pour H. Stern, les réparations visibles sur la mosaïque no 58 (en quadrillage droit, et assez proche de notre mosaïque Guilhermet par ses remplissages géométriques) sont "probablement de la même main que celles du $n^{0} 61$ ". En admettant que l'ensemble des pavements du "Verbe incarné" (nos 53 à 63) soit bien homogène, comme l'affirme II. Stern, la fin clu II e siècle et l'incendie de 197 pourraient ronstituer, pour Lyon, un lerminus analogue à celui de l'inondation des quartiers riverains du Rhône à Vienne.

Si ces recoupements étaient fondés ${ }^{49}$, on aurait là te moyen de rapprocher la production de Vienne et celle de Jyon qui, à tant d'egards, mais surtout pour ces deux types de trames, apparaissent lies par des rapports etroits, au point que H. Stern n'hésitait pas à «attribuer à un mème atelier, sans doute fixé à Vienne » les pavements nos $53.3,58,59$ de Lyon et les numéros 158,198 et 200 de Sainte-Colombero. La mosaïque Guilhermet, appartenant à cette série, se trouverait ainsi datée anant la fin du $\mathrm{II}^{\mathrm{e}}$ siècle.

Cette thérorie, relativement satisfaisante pour l'esprit, si elle ne vise qu'à proposer un lerminus post quem à ret ensemble de mosiäques, est beaucoup plus contestable lorsqu'il s'agit de fixer le lorminus anle quem et de faire croluer les deux séries de trames l'une par rapport à l'autre, comme le fait J. Lancha. L Lauleur. en effet, commenee par arlmettre un postulat éminemment contestable et qui est le "décalage d'un demi-siècle environ existant entre l'utilisation d'un canevas en ltalie of sa forme provinciale». A partir de cette prémisse gratuite, elle fixe à 150 après J.-C. la date avant laquelle le quadrillage de bandes croisées de la mosiäque $n^{0} 173$ de Vienne ne saurait avoir etée exécuté. A supposer même que l'on acepte ce postulat, la sureession quadrillage droit chargé d'un décor multiple, puis, après 150, quadrillage de bandes croisées, ne s'impose pas pour autant.

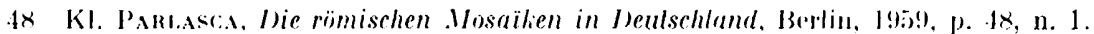

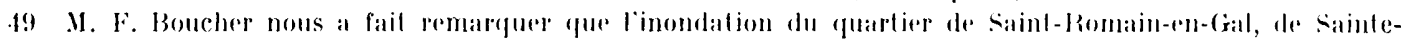

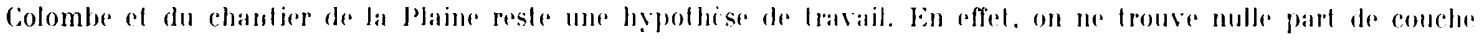

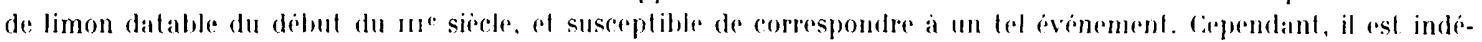

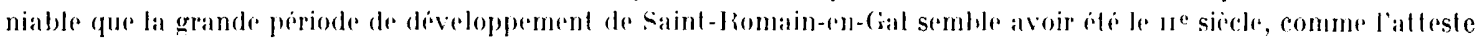

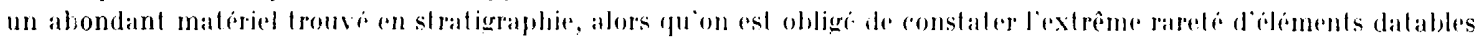

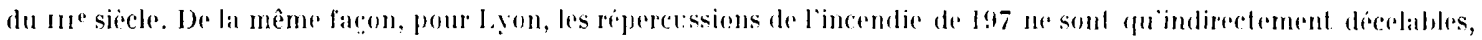

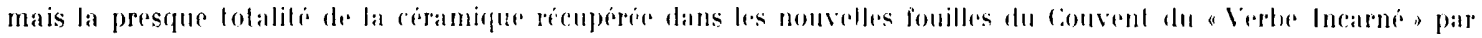

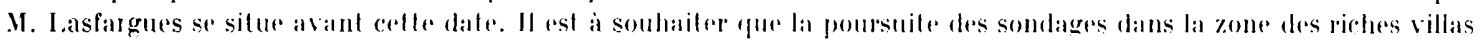

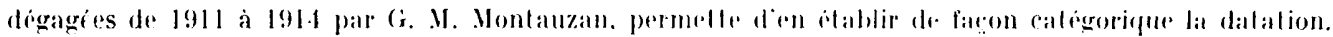

5) H. STrkx, dans . Mon. Piol, cil., p. 1?. 
In détail du décor des quadrillages sur lequel l'altention n’a pas été suffisamment attirée, et qui est la présence des bordures intérieures, inviterait plutôt à considérer que ces deux variantes d'une mème trame se développent parallèlement.

On s'accorde à penser que dans le répertoire du décor multiple. les bordures intérieures de chaque panneau (qui peuvent revêtir les formes de lignes de carrés sur la pointe, lignes de triangles superposés, (hevrons. ete.) sont les restes. ou les organes-témoins de lrur origine italique, que l'on doit chercher dans les mosaïques à caissons de l'époque républicaine et proto-augustéenne. Le meilleur exemple, en Gaule, en est la grande mosaïque d'Orangers a placer dans la première moitié du I $^{\text {er }}$ siècle. La dérivation des mosaïques "a cassettoni " est encore très evidente : les petits panneaux qui composent le quardrillage ont chacun une bordure intérieure et un fleuron simplifié, qui s'enlève sur fond noir. rappelant le décor des plafonds à lacunaria de Pompéi.

Quant à lutilisation dans la vallée du Rhòne du schéma à quadrillage de bandes croisées, clle apparait bien contemporaine et non postérieure. On en a un témoignage dans un fragment trouvè à Aix-en-I'rovencesa, daté du début du er siècle par les fouilleurs, dans un pavement d'Orange ${ }^{53}$ probablement du milieu du I $^{\text {er }}$ siècle, et dans une mosaïque mutilée de Vaison-la-Romaine, probablement du début du II ${ }^{\mathrm{e}}$ siècle ${ }^{54}$. Dans ces trois exemples, les panneaux du quadrillage ne comportent aucune bordure intérieure, comme c’était déjà le cas dans les mosaïques italiques dont ils reprennent les schémas, et certains remplissages géométriques simples ${ }^{55}$. Mème dans la partie Nord de la Gaule, la composition en quadrillage de bandes croisées apparaît à époque haute sous une forme tres proche ${ }^{56}$.

Le premier cas qui montre que les deux schémas, quadrillage droit et quadrillage de bandes eroisées se còtoient et s'empruntent réciproquement leur répertoire décoratif nous semble être un pavement de Vienne ${ }^{57}$, aujourd'hui au Musée Saint-Pierre. Son caractère hybride se traduit précisément par la présence de petits caissons carrés ornementés intérieurement d'une ligne de triangles superposés. Or, cette mosaïque a toutes les caractéristiques d'un pavement d'époque ancienne : finesse de la technique, sobriété de la polychromie (noir, rouge, blane, ot un jaune seulement dans la tresse), austérité des remplissages géométrifues (motifs emboités dans les losanges et les petits carrés d'intersection), simplicité des fleurons dans des caissons de dimensions très réduites (identiques à ceux de la mosaïque d'Orange, et sur fond noir), aspect très classique de l'emblema central (tête de vieillard, ou masque, dans un bouclier d'écailles bi-partites noires et blanches), schématisme du rinceau à boules qui rappelle les exemples particulièrement grêles de

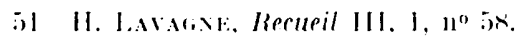

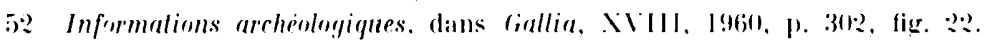

5.3 Recueil III. I, $\mathrm{n}^{\circ}$.5.).

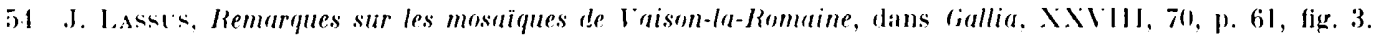

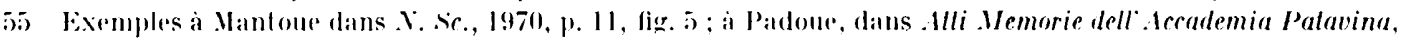

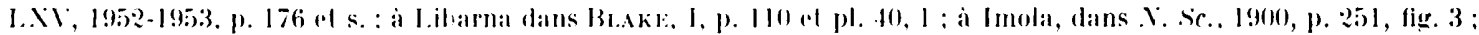

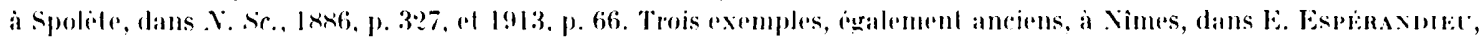

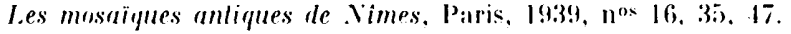

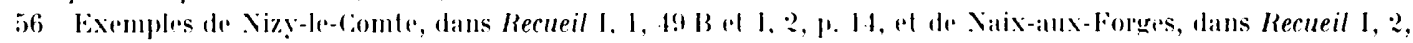

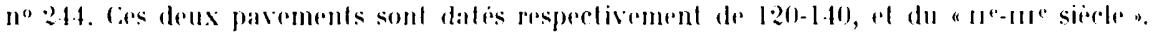

57 Ims. Mos. I, no $19(1)$. 
Pompéi. l'ensemble etant ceinturé par une large bordure noire; tous les traits en font indiscutablement une mosaïque ancienne of non pas. comme le propose J. Ianchas, un point d'aboutissement. Yous la daterions volontiers des années 120-140, comme la mosaïque de Nizy-le-Comte dont elle reprend le motif (rare), de l'étoile à quatre pointes rhargée d'un carré concave.

De la même façon, et à peu près contemporaine, serait à placer la mosaïque d'Anse ${ }^{5 x}$, où l'on voit dans la bordure alterner les caissons les plus traditionnels entourés intérieurement de lignes de triangles superposés, de lignes de carrés se touchant par la pointe. de chevrons, tandis que le champ lui-mème est constitué par les cases d'un quadrillage à bandes croisées où figurent des remplissages de type classique notamment la roue tournoyante ${ }^{59}$, ou les scula qui figurent sur les pavements et les plafonds d'époque républicaine. les autres éléments décoratifs confirment cette impression d'ancienneté, tout particulièrement la bordure en proues de navires qui apparaît précisément dans un parrement à caissons de Rome, daté du milieu du $\mathrm{I}^{\mathrm{er}}$ siècle avant J.-C. ${ }^{60}$, ou encore le motif cordiforme de la seconde bordure interieure assez proche d'un exemple pompéien ${ }^{61}$, et d'une bordure d'une mosaïque de Saint-Paul-Trois-Châteaux ${ }^{62}$, que nous datons du $\mathrm{e}^{\text {er }}$ siècle. La mosaïque d'Anse ne nous parait done pas devoir être datée du troisième quart du we siècle encore moins de l'époque sévérienne ${ }^{63}$, mais bien du début du $\mathrm{I}^{\mathrm{e}}$ siècle. comme on l'avail proposé lors de la première publication ${ }^{64}$.

Ainsi les mosaïques nos 190 de Vienne et 17i) d'Anse ne marquent pas, selon nous. un "achèvement", ou la victoire du schéma à quadrillage de bandes croisées sur le quadrillage droit dont il conserverait quelques traces; elles constituent, au contraire, Ir témoignage d'un moment rharnière de l’évolution où les deux trames qui se sont développées parallèlement finissent par se rejoindre an puisant l'une et l'autre à une mème source, qui est le decor multiple. Celui-ci n'est pas lié à un quadrillage particulier ${ }^{65}$. mais offre seulement un répertoire commode pour les ateliers qui, au début du II $^{\mathrm{e}}$ siècle. utilisent concuremment le quadrillage droit et le quardrillage de bandes croisées.

On n'en voudra pour preuve que la grande mosilique du "Verbe Incarné ${ }^{66}$, très justement rapprochée de la mosaïque d'Anse, jusqu'à proposer qu'elle soit de la "mème époque et du même atelier ${ }^{67}$. On ajoutera à ce rapprochement, celui de la mosiäque no 190 de Vienne. En effet, dans cette dernière, on note que les caissons sont presque identiques aux trois premiers pannealux carrés de la mosaïque du "Verbe Incarnè» (rangéés extérieures

5x Rerueil II, 3, $\mathrm{n}^{\circ} 1 \%$ \%. $11^{\circ}+46$

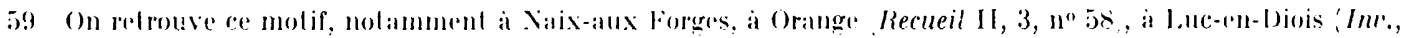

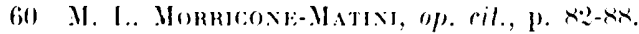

(i) P'serick, op. cil., pl. 50, 4.

(i2) Recueil III, 1, no 103.

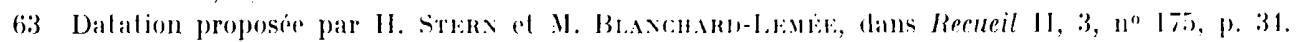

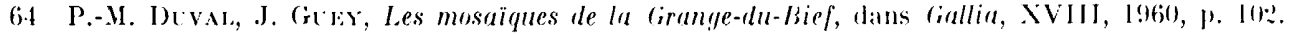

65 On le trouve dans des compositions en eloiles de huil losanges par exemple a Besaneon, dans Rerueil J, 3,

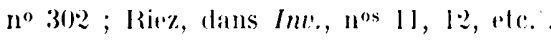

fif lion, Recueil II, 1, $\mathrm{n}^{\circ} 53$.

bi. (i. 11. (i.3. 
a. e. $i$ ). le quatrième caisson (aisson $h$ ) apparaissant dans la seconde mosaïque d'Anse68. si on ne considérait que les rangées extérieures de la mosaïque lyonnaise. nul doute que la répétition des motifs géométriques qui remplissent les cases carrées rectangles of losanges étant simplement emboités) norienterait la comparaison avec des mosaïques du type de celle de Vienne no 1911). Au contraire. à ne prendre en comple que les rangées intérieures de ce pavement. on serait tenté de ne penser qu à la variété des remplissages lans les mosiäques viennoises les plus élaborées. ou encore aux motifs couvrants des parerments en quadrillage droit. tels que les mosilïguess Séguin. Michourl, ou l'Achille à Skingres de Sainte-Colombe. Examinons les motifs couvrants des carrés $c$, d. g. z. de la mosiäque du "Verbe Incarne» : on constate que ce sont ceux des mosiïques à quadrillage précedemment cités. cl tout particulièrement reux de notre mosaïque Guilhermet (c. d. g. z, y figurent).

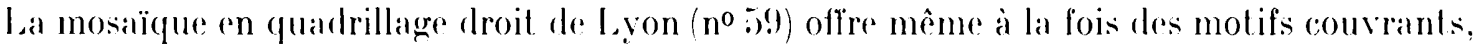
traditionnels dans cette trame. al deux panneaux carrés divisés en quatre éléments (panneau $g$, et $p$ ) qui regroupent, comme en une sorte de compendium des motifs de quadrillage de bandes croisées, et notamment les trois premiers caissons de la mosiäque lyonnaise $n^{0}$ 53. On voit par cette intrication des thèmes ornementaux et ces multiples rappels de motifs, combien les deux séries de trames sont liées dans leur développement. sans qüil soit possible de distinguer une suecession chronologique dans leur apparition ret leur vogue. On peut seulement tirer argument de la plus ou moins grande fréquener des emprunts dans les remplissages qui chargent l'une et l'autre pour en déduire que les pavements les plus composites dans leur décor ont toutes chances d'être les plus tardifs, c'est-à-dire de la fin du ri $^{\mathrm{e}}$ siècle. tels la grande mosäque du "Verbe Incarné ». ou le pavement no j!l qui était placé à côté d'elle. La mosiäque Guilhermet, comme la mosäque d'Achille à Skypros, ou le parement Michoud, par leur registre un peu moins étendu, sont probablement légèrement antérieures.

La conclusion qui nous parait s̈imposer est qüil est vain de vouloir discerner une crolution chronologique dans l'utilisation de ces deux trames a l'intérieur du II $^{\mathrm{e}}$ siècle. La confrontation des exemples de Lyon at de Vienne est suffisamment claire en elle-même. Dans les deux villes les plus fécondes en découvertes de mosaïques. il se trouve que nous avons deux domus dans lesquelles voisinent des parements représentant des exemples lypiques de canevas en quadrillage droit et de trames en quadrillages de bandes croisées. Pourquoi se refuser à les considérer comme contemporains of ne pas y voir le reflet de deux courants artistiques également à la mode? Les liens datelier entre Vienne et Lyon apparaissent si nombreux qu'il n'est pas étonnant de trouver deux schémas de constructions très voisins, objets d'une mème vogue et à la mème époque. dans deux cités très proches.

Jenti LaVAGiNe. 